\title{
Commentary: Another exercise in the educational gymnasium
}

\author{
Edward D. Verrier, MD
}

\author{
From the Department of Cardiovascular Surgery, University of Washington, Seattle, Wash. \\ Disclosures: Author has nothing to disclose with regard to commercial support. \\ Received for publication June 3, 2019; accepted for publication June 3, 2019; available ahead of print July 10, \\ 2019. \\ Address for reprints: Edward D. Verrier, MD, K Alvin Merendino Professor of Cardiovascular Surgery, University \\ of Washington, 1959 NE Pacific St, Seattle, WA 98195 (E-mail: edver@u.washington.edu). \\ J Thorac Cardiovasc Surg 2020;159:1445-6 \\ $0022-5223 / \$ 36.00$ \\ Copyright (C) 2019 by The American Association for Thoracic Surgery \\ https://doi.org/10.1016/j.jtcvs.2019.06.007
}

So, now we have evidence of concept that that the certifying board examinations in cardiothoracic surgery can be simulated with accuracy in a single institution committed to surgical education (University of Texas MD Anderson Cancer Center, in Houston, Tex). The conclusions of the article in this issue of the Journal from Corsini and colleagues, ${ }^{1}$ titled "Cardiothoracic Surgery Mock Oral Examinations: A Single Institution's 5-Year Experience,' are basically 3-fold: (1) mock oral examinations can be accurately simulated in a single institution; (2) organizing such a yearly effort takes significant administrative and faculty effort, including both educational time and logistic organization; and (3) such an exercise may be a useful adjunct assessment tool in the armamentarium for resident evaluation.

Let us start with some assumptions on why residents perform poorly on the oral examination, recognizing that the goal of the examination is to make sure that practicing surgeons are safe and competent. Most cardiothoracic surgical resident trainees have been carefully vetted for intellectual capacity, clarity of thought process, and work ethic, starting early in their educational preparation and continuing through medical school and residency training. They have been recommended by their program director and faculty after many years of intense training and observation to be ready and competent to sit for the examination. The board questions themselves have gone through an exhaustive vetting process to be fair and representative of everyday practice in the broad specialty of cardiothoracic surgery. Having said all that, certainly there is a small percentage of applicants who have leaked through the cracks and who are not qualified to do all aspects of cardiothoracic surgery and thus should not be certified as meeting American Board of Thoracic Surgery standards. Another potential reason that applicants may not pass 1 portion of the 3 part examination is that they focused too early and therefore have failed to prepare properly for one or more aspects of the training or examination. There obviously could be language, cultural, or presentation style challenges, with the

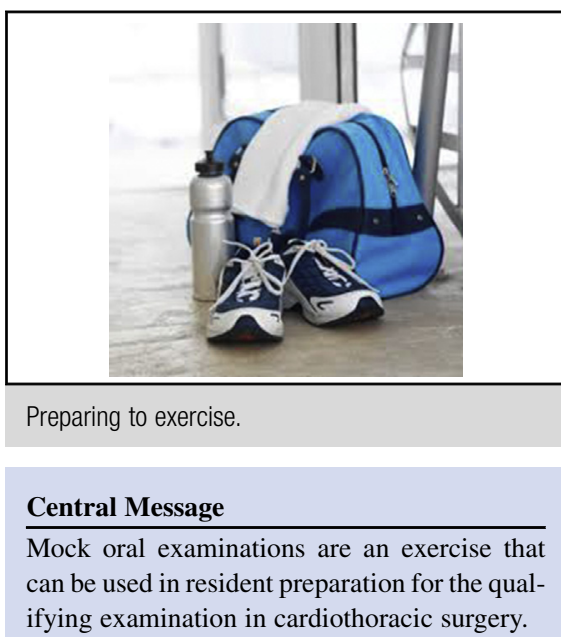

See Article page 1439. potential for implicit bias on the part of either the examiner or examinee that could affect the process. In addition, however, because this is high stakes examination, performance anxiety is real. Most of us who have been program directors for years have had residents go into the process who we might have thought were among the best of our trainees but did not pass this oral examination, and when debriefed, they state that they simply froze.

As surgical educators, every day in the classroom, at the bedside, or in the operating room, we ought to be role modeling and training our residents in how to listen carefully to patient histories, do the proper examination, order and interpret the right diagnostic testing, develop judgment, formulate the optimal plan, and execute the highest quality operation-which is exactly the process that this examination is trying to evaluate-so, therefore, why would anyone want to invest the faculty and administrative resources to simulate for this single test? It must have something to do with the perceived value of rehearsal, warm-up, and deliberate practice. ${ }^{2}$ First, we know that these examinations have little to do with long-term success as a surgeon and may be fraught with issues of validity and interobserver variability. ${ }^{3}$ We know that cramming can improve scoring on short-term memory recall, which is the basis of the multiple-choice questions used for the qualifying examination, ${ }^{4}$ but it also is not a substitute for learning. ${ }^{5}$ In contrast, one might think it important that a surgeon be measured for technical skill quality; however, we do not measure 
technical skills, because we have not figured out a practical way to do this. ${ }^{6}$

The multiple-choice examination for fund of knowledge recall and the oral qualifying exam for decision making and judgment are ingrained in our assessment culture, so they must be conquered by our successful trainees. So, similar to the way that "cramming our short-term memory" may have a positive short-term influence on the qualifying examination results, "mock oral examinations" may be a useful exercise in the preparatory gymnasium for the certifying examination. Minimizing variables such as presentation, implicit bias, lack of examination taking rehearsal or preparation, and performance anxiety are all desirable outcomes of preparation, because those variables do not reflect on surgeon safety, nor do they reflect the true competence of a surgeon day in and day out. Mock oral examinations cannot be a substitute for resident or lifetime learning, but they may be an assessment exercise in the educational gymnasium.

\section{References}

1. Corsini EM, Mitchell KG, Nguyen TC, Vaporciyan AA, Antonoff MB. Cardiothoracic surgery mock oral examinations: a single institution's 5-year experience. $J$ Thorac Cardiovasc Surg. 2020;159:1439-44.

2. Verrier ED. The elite athlete, the master surgeon. J Am Coll Surg. 2017;224: 225-34.

3. Schubert A, Tetzlaff JE, Tan M, Ryckman JV, Mascha E. Consistency, inter-rater variability, and validity of 441 consecutive mock oral examinations in anesthesiology: implications for use as a tool for assessment of residents. Anesthesiology. 1999; $91: 288-98$.

4. Luc GY, Verrier ED, Allen MS, Aloia L, Baker C, Fann JI, et al. Does cramming work? Impact of National Web-Based Thoracic Surgery Curriculum login frequency on thoracic surgery in-training exam performance. J Thorac Cardiovasc Surg. 2018;156:922-7.

5. Oyentunji SO, Mokadam NA. Cramming is not learning. J Thorac Cardiovasc Surg. 2018;156:928.

6. Moorthy K, Munz Y, Sarker SK, Darzi A. Objective assessment of technical skills in surgery. BMJ. 2003;327:1032-8. 